\title{
Fast and Ecological Liquid-Liquid Separation Method for Preparing Quinones Enriched Extract from Nigella sativa Oil
}

\author{
Klara Urbanova, ${ }^{a}$ Pavel Kloucek, ${ }^{b}$ Jaroslav Havlik, ${ }^{b}$ Irena Valterova ${ }^{c}$ and \\ Ladislav Kokoska ${ }^{\oplus *, d}$ \\ ${ }^{a}$ Department of Sustainable Technologies, Faculty of Tropical AgriSciences, \\ Czech University of Life Sciences Prague, Kamycka 129, 16521 Prague 6-Suchdol, \\ Prague, Czech Republic \\ ${ }^{b}$ Department of Quality of Agricultural Products, Faculty of Agrobiology, \\ Food and Natural Resources, Czech University of Life Sciences Prague, Kamycka 129, \\ 16521 Prague 6-Suchdol, Prague, Czech Republic \\ ${ }^{c}$ Institute of Organic Chemistry and Biochemistry, Academy of Sciences of the Czech Republic, \\ Flemingovo No. 2, 16610 Prague 6, Prague, Czech Republic \\ ${ }^{d}$ Department of Crop Sciences and Agroforestry, Faculty of Tropical AgriSciences, \\ Czech University of Life Sciences Prague, Kamycka 129, 16521 Prague 6-Suchdol, \\ Prague, Czech Republic
}

\begin{abstract}
A fast, inexpensive, and ecological method based on liquid-liquid extraction principle has been developed for preparation of extract with high content of Nigella sativa ( N. sativa) quinones. The gas chromatography-mass spectrometry (GC-MS) analysis showed that oil extraction using $70 \%$ ethanol for $6 \mathrm{~h}$ yielded extract containing $50 \%$ of thymoquinone (TQ) and $13 \%$ of thymohydroquinone (THQ).
\end{abstract}

Keywords: Nigella sativa, quinones, crude oil, liquid-liquid extraction

\section{Introduction}

Since the more environmentally friendly and selective techniques are nowadays preferred for natural products extraction, the development of new designs of green and sustainable methods of separation is currently a hot research topic in the multidisciplinary area of applied chemistry, biology, and technology. The principles of green extraction can be generalized as the discovery and the design of extraction processes, which could reduce the energy consumption, allow the use of alternative solvents and renewable/innovatory plant resources to eliminate petroleum-based solvents and ensure safe and high quality extracts or products. ${ }^{1}$ Among them, techniques requiring expensive and highly sophisticated equipment such as supercritical fluid extraction (SFE) and pressurized liquid extraction have recently received attention. ${ }^{2}$ However, conventional approaches such as liquid-liquid extraction

*e-mail: kokoska@ftz.czu.cz using non-toxic solvents are remaining widely employed and are considered useful techniques, because they are simple, fast, reasonably selective and do not require expensive equipment. The development of new methods satisfying the conditions of green extraction of natural products using conventional methods is therefore still needed, especially for the processing of natural materials with pharmacological and health beneficial effects such as herbal drugs. ${ }^{3}$

Nigella sativa ( $N$. sativa) is an annual plant belonging to the Ranunculaceae family, native to the Mediterranean region and the Middle East, whose seeds have traditionally been used in the Indian subcontinent, Arabian countries, and Europe for culinary and medicinal purposes. ${ }^{4}$ They contain between 25 and $40 \%$ of crude oil consisting mainly of mono-, di- and triacylglycerols, fatty acids (linoleic, oleic, palmitic, stearic) and sterols. ${ }^{5}$ Thymoquinone (TQ), one of the major biological active compounds of the seeds, is present in various amounts in both crude and essential oils. ${ }^{6}$ It has shown cardio-, gastro-, hepato-, nephro- and neuroprotective action as well as antiarthritic, antihyperglycemic, 
anti-inflammatory, antimicrobial, antioxidative, antitumor and immunomodulatory effects in various in vitro and in vivo systems. TQ has also demonstrated very low grade of toxicity. ${ }^{7}$ In previous clinical studies, TQ showed antiepileptic action in children and its intracrevicular application has been found to be beneficial in treating chronic periodontitis. ${ }^{8,9}$ The reduced form of TQ, thymohydroquinone (THQ), is also considered as perspective biological active compound with antibacterial, antifungal, anti-inflammatory, antioxidative, and antitumor effects. ${ }^{10,11}$ The inhibitory activity for acetylcholinesterase was also observed in this substance. ${ }^{12}$

As it has previously been shown in several experiments, the content of TQ and total chemical composition of $N$. sativa seed extracts, crude and essential oils are significantly influenced by the isolation method used. Hydrodistillation by Clevenger-type apparatus and simultaneous steam distillation-extraction using Likens and Nickerson's apparatus are traditional, relatively easy and cheap approaches; however, their disadvantages are long extraction time, thermal degradation and a low content of TQ in final product varying from 0.06 to $24.6 \%{ }^{13}$ Extraction with organic solvents (e.g. acetone, ether, chloroform, hexane, methanol, petroleum ether) using a Soxhlet extractor often combined with subsequent steam distillation of the extract is another frequently used approach. ${ }^{14,15}$ In our previous study ${ }_{1}^{16}$ we used a combination of extraction with hexane in the Soxhlet apparatus followed by re-extraction with methanol for determination of TQ in different plant materials. Advantage of these methods is the higher efficiency, but disadvantages are higher cost and the use of toxic solvents. One of the most efficient methods is supercritical fluid extraction providing extracts with high content of TQ (79-86\%). ${ }^{17}$ Despite the fact that experiments comparing efficacy of hydrodistillation, steam distillation, steam distillation of the oils obtained by Soxhlet extraction by an organic solvent and extraction by supercritical carbon dioxide confirmed the highest efficacy of the SFE, the significant disadvantages of extraction using supercritical fluid are very high costs for acquisition and operation of extraction equipment. ${ }^{14}$ Therefore, we developed a fast and effective liquid-liquid separation method for preparation of extract with enriched content of TQ from the $N$. sativa oil that does not require expensive equipment, does not use toxic solvents and is timesaving.

\section{Experimental}

\section{Oils and chemicals}

Three different samples of cold pressed $N$. sativa oils were obtained from commercial suppliers: A: Saloos naturcosmetic (Breclav, Czech Republic), B: Oleador (Hannover, Germany), and C: Dary zeme (DobrichovPecky, Czech Republic). Ethanol (96\% p.a.) was purchased from Lach-ner (Neratovice, Czech Republic). Analytical standards (2,4-diethoxybenzoic acid; 2,4-decadienal; 2-decenal; 3,7-dimethyloctanal; 4-terpineol; dihydrocarvone; hexadecanoic acid; limonene; octadecadienoic acid; octadecenoic acid; $p$-cymene; sabinene; tetradecane; thymol; TQ; $\alpha$-pinene; $\beta$-pinene; $\gamma$-terpinene), $n$-alkanes series ranging from $\mathrm{C} 9$ to $\mathrm{C} 29$ and 1-bromdecane were supplied by Sigma-Aldrich (Prague, Czech Republic). The THQ analytical standard was prepared according to the procedure previously described by Tesarova et al. ${ }^{11}$

\section{Extraction procedure and conditions}

In the first experiment, $1 \mathrm{~mL}$ of $N$. sativa oil was mixed with $1 \mathrm{~mL}$ of $30,40,50,60,70,80$, and $90 \%$ solution (v/v) of ethanol and deionized water obtained from an Aqal 29 system (Brno, Czech Republic). The mixture was shaken in $4 \mathrm{~mL}$ glass vials (Agilent Technologies, Santa Clara, USA) on a laboratory shaker (IKA Vortex 2, Staufen, Germany) at $2000 \mathrm{rpm}$, at $21{ }^{\circ} \mathrm{C}$ for $24 \mathrm{~h}$. After $12 \mathrm{~h}$ of equilibrium, two easily separable layers emerged (oil and hydroalcoholic phases), which were separated by Pasteur pipette. The chemical compositions of both oil and ethanol/water phases were immediately analyzed by gas chromatography-mass spectrometry (GC-MS).

As a second experiment, the hydroalcoholic layers were periodically taken and analyzed after 2, 4, 6, 8, 10, 12 and $24 \mathrm{~h}$ with the aim to determine optimum extraction time. The $70 \%$ ethanol was used as extraction solvent. In the third experiment, three different oils (A, B and C) were extracted with $70 \%$ ethanol for six hours and investigated by GC-MS, with the aim to determine influence of variability of extraction matrices on chemical composition of the extract. The chemical compositions of oil samples were determined by GC-MS prior extraction. All other extraction conditions of experiments 2 and 3 were identical with those of the first experiment. Each experiment was performed in three independent replicates.

\section{GC-MS analysis}

Analyses were carried out using a Thermo Scientific Focus GC with DSQ II MS (Thermo Fisher Scientific, Waltham, USA). Separation was achieved on a fused-silica DB-5 column ( $30 \mathrm{~m} \times 0.25 \mathrm{~mm}$, film thickness $0.25 \mathrm{~mm}$, J\&W Scientific, Folsom, USA). The samples of the raw oils and oily phases remaining after extraction were dissolved in hexane (20 mg of oil to $1 \mathrm{~mL}$ ) and injected in splitless 
mode, injection volume being $1 \mu \mathrm{L}$. The hydroalcoholic samples were injected directly. 1-Bromdecane was added to the samples as an internal standard for quantification $(0.1 \mu \mathrm{g}$ per $1 \mu \mathrm{L}$ of the extract). The content of TQ and THQ is presented as internal standardization (IS) value (ratio of peak area to internal standard) with standard deviation. The percentage composition of raw and extracted essential oil were computed from electronic integration of GC peak areas. All compounds for which relative peak areas were recorded as less than $0.1 \%$ in all samples were excluded from the analysis. ${ }^{18}$ The operating conditions were as follows: injector temperature, $200{ }^{\circ} \mathrm{C}$; carrier gas, He $\left(1 \mathrm{~mL} \mathrm{~min}^{-1}\right)$; oven temperature program, $60{ }^{\circ} \mathrm{C}$ (2 min) to $200{ }^{\circ} \mathrm{C}$ at a rate of $3{ }^{\circ} \mathrm{C} \mathrm{min}^{-1}$, then to $310^{\circ} \mathrm{C}$ at $25{ }^{\circ} \mathrm{C} \mathrm{min}{ }^{-1}$ and held isothermal for $10 \mathrm{~min}$. The data processing was performed by Excalibur software (Thermo Fisher Scientific, Waltham, USA). A mass detector was set to the ionization energy, $70 \mathrm{eV}$; ion source temperature, $200{ }^{\circ} \mathrm{C}$; scan time, $1 \mathrm{~s}$; mass range, 30-600 amu. The identification of the chemical constituents was based on the co-injection of authentic compounds (standards), on the comparison of their Kovats retention indices and retention time, and on the matching of mass spectra against those listed in commercial libraries (National Institute of Standards and Technology Library, ver. 2.0.f).

\section{Results and Discussion}

In this study, a fast, inexpensive and ecological liquidliquid separation method for preparing THQ and TQ enriched extract from $N$. sativa seed oil was developed in several steps, which include extraction of the oil with the solution ethanol-water, determination of the most suitable concentration of ethanol and the best extraction time on quinones recovery as well as chemical analyses of initial materials (crude oils) and extraction products (enriched extracts).

Effect of ethanol concentration and extraction time on quinones recovery

At first, the $N$. sativa oil (sample A) was extracted with ethanol at concentrations varying from 30 to $90 \%$. Consequently, two separate liquid layers were formed, and the top layer was enriched with TQ and THQ. Figure 1 demonstrates a gradual increase of content of TQ in extract, when the maximum was obtained using $60-80 \%$ ethanol. At higher concentration of ethanol (90\%), the content of extracted TQ decreased. Despite minimal differences in the contents of TQ varying for 60,70 and $80 \%$ of ethanol from $4.69 \pm 0.27$ to $5.14 \pm 0.35$ of IS value, concentration $70 \%$ of ethanol has been chosen as the most efficient for its extraction. In case of THQ, the extraction was most efficient using 50 and $60 \%$ ethanol with $6.18 \pm 0.16$ and $6.43 \pm 0.49$ of IS values, respectively (Figure 1 ).

The increase of the content of quinones is due to their different solubility in the solutions of different concentration of ethanol in water. Since the water solubility of $p$-benzoquinones is generally lower than that of their reduced forms, ${ }^{19}$ this feature may explain the difference in optimal solvent concentration for TQ and THQ extraction. The reduction of TQ to THQ during the extraction process can also contribute to the increased amount of THQ in $40-60 \%$ ethanol extracts. ${ }^{20}$ In contrast, the remaining amount of TQ and THQ in the oil after extraction was about $1-2 \%$ confirming its high efficiency. Different ratio

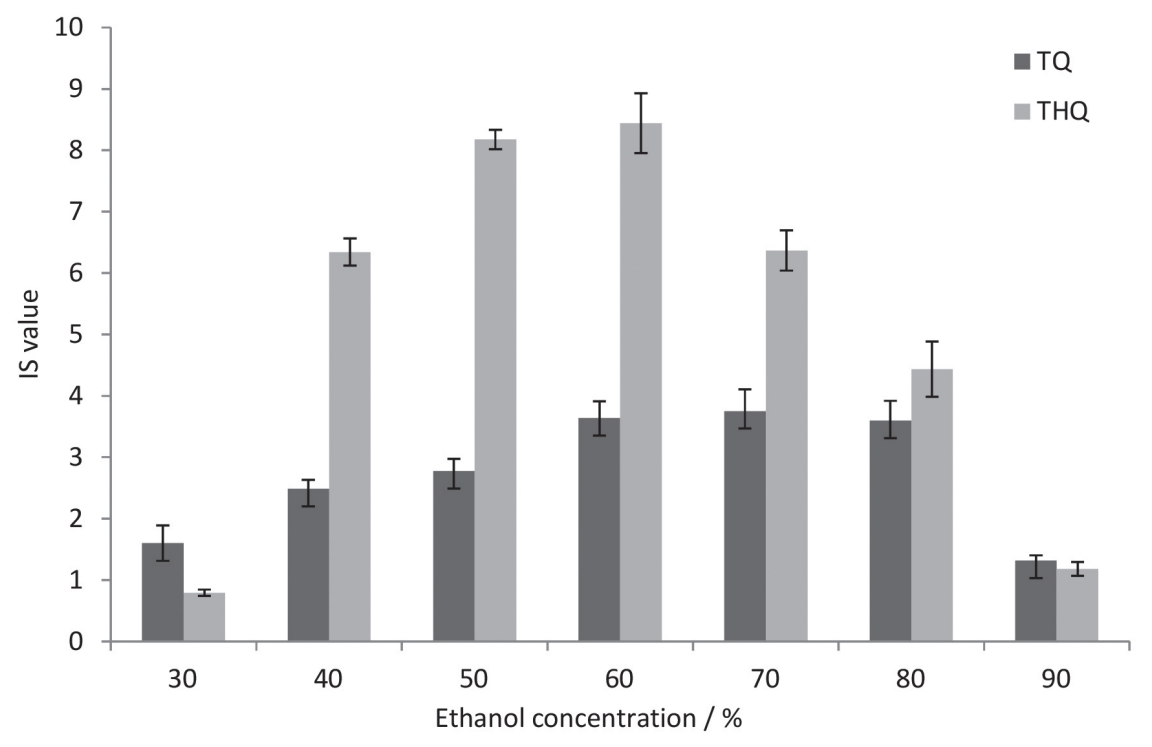

Figure 1. Effect of ethanol concentration on thymoquinone (TQ) and thymohydroquinone (THQ) extraction. 
ethanol-water was already used for extraction of phenolic compounds from Salvia officinalis, whereas the optimal hydroalcoholic solvent composition was between 55 and $75 \%(\mathrm{~m} / \mathrm{m})$ ethanol. ${ }^{21}$ In another study, ${ }^{22}$ phenolic compounds were successfully extracted using $60 \%$ aqueous mixtures with ethanol, methanol and acetone. According to our best knowledge, this is the first report describing extraction of quinones using ethanol-water mixture.

The extraction time is essential in economizing energy and cost of the extraction process. Therefore, the effect of the extraction time on the efficiency of the process was monitored for both TQ and THQ as a second step of our experiments. Figure 2 shows that the effectiveness of quinones recovery was observed in time-dependent manner.

Almost no quinones passed into the hydroalcoholic layer after two hours of extraction, whereas after $4 \mathrm{~h}$ their recovery was maximal. After $6 \mathrm{~h}$, the oil still contained some amount of both compounds, but the effectivity of the extraction process significantly decreased. Finally, the most effective time of extraction of quinones was determined as $4 \mathrm{~h}$. This is in correspondence with previous reports ${ }^{21-24}$ showing that the extraction time between $3-4.5 \mathrm{~h}$ is optimal for isolation of phenolic compounds, because after that time the total phenolics recovery stayed constant and longer extraction times increase the possibility of phenolics oxidation.

Influence of extraction matrices on qualitative and quantitative parameters of extracts

With the aim to determine influence of variability of extraction matrices on chemical composition of the extract, three different $N$. sativa crude oils (A, B and C) were extracted with $70 \%$ ethanol for six hours and investigated by GC-MS. The analysis of all three oils performed prior the extraction showed presence of 23 volatile compounds (Table 1). Octadecadienoic and octadecenoic acids (32.86 and $21.63 \%)$, followed by $p$-cymene $(11.55 \%)$ and TQ $(9.37 \%)$ were identified as main components of the crude oil A. The major constituents of the oils B and C were TQ (32.95 and 39.73\%) and $p$-cymene (17.97 and 27.74\%). As reported earlier, the differences in the contents of individual components between different types of oil may be caused by different origin of $N$. sativa seeds. ${ }^{25,26}$ In addition, $N$. sativa crude oil contains also significant amounts of triacylglycerols, sterols and phospholipids. ${ }^{27}$ Since these compounds cannot be detected by GC-MS without previous modification, they can also be present in analyzed oil samples as well in the final extract.

The results of extraction procedure showed the presence of 17 components in the all three extracts obtained from crude oils A, B, and C (Table 1). All these extracts contained mainly TQ (29.83, 47.39 and 50.62\%) and THQ (13.65, 10.80 and $13.12 \%$ ), followed by significantly lower amounts of terpenes such as $p$-cymene $(7.28,3.50$ and $7.59 \%)$, trans-4-methoxythujane $(6.55,3.71$ and $4.81 \%)$, thymol $(2.76,1.59$ and $4.82 \%)$ and $\alpha$-thujene (2.78, 1.36 and $1.42 \%)$. Although there are significant differences in the contents of fatty acids in the raw oil samples ranging from 0.91 to $32.86 \%$, the variances are minor after extraction (0.26-6.64\%). It is therefore evident that most of fatty acids remain in the oil phase. Despite these differences, the described extraction method is effective for all types of tested oils.

Some of the compounds detected in the fraction such as $p$-cymene, thymol and $\alpha$-thujene have previously shown various biological activities (e.g. antioxidant and

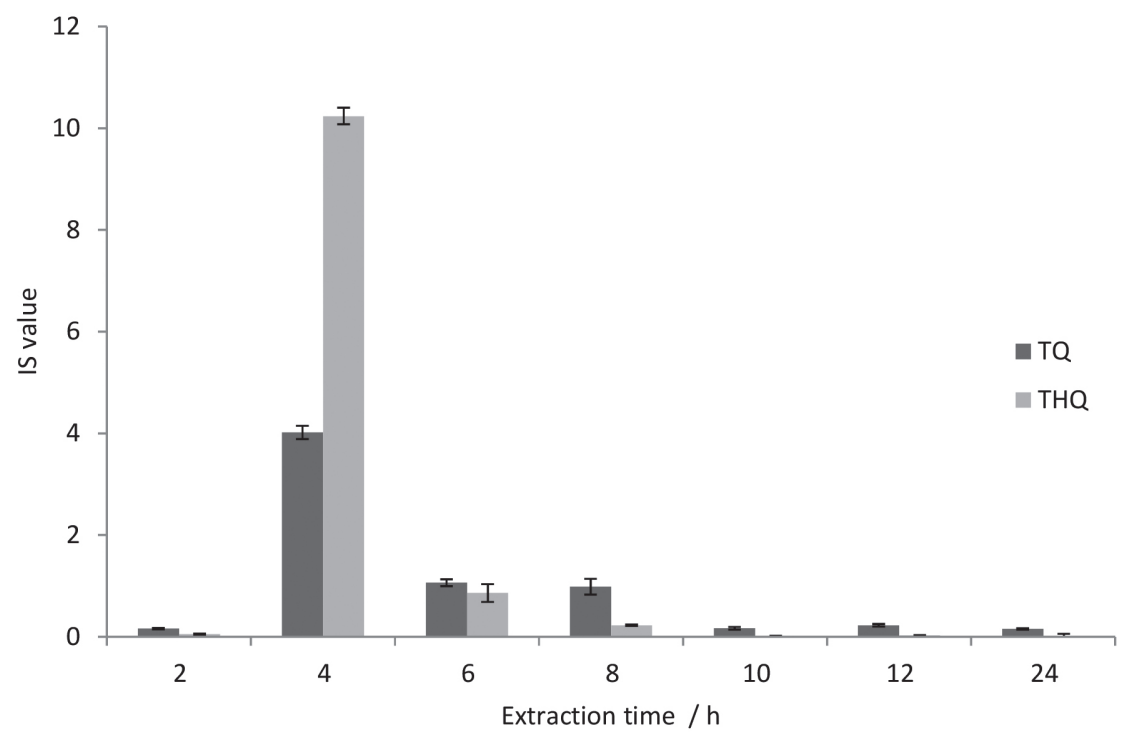

Figure 2. Effect of time on the extraction of thymoquinone (TQ) and thymohydroquinone (THQ). 
Table 1. Composition of extracts obtained by $70 \%$ ethanol from crude oils of $N$. sativa seeds

\begin{tabular}{|c|c|c|c|c|c|c|c|c|c|c|}
\hline \multirow{2}{*}{ No. } & \multirow{2}{*}{ Compound } & \multicolumn{2}{|c|}{ Retention index } & \multicolumn{3}{|c|}{ Oil (peak area) / \% } & \multicolumn{3}{|c|}{ Extract (peak area) / \% } & \multirow[t]{2}{*}{ Identification $^{\mathrm{c}}$} \\
\hline & & Observed $^{\mathrm{a}}$ & Published $^{\mathrm{b}}$ & A & B & $\mathrm{C}$ & A & B & $\mathrm{C}$ & \\
\hline 1 & $\alpha$-thujene & 938 & 931 & 4.91 & 10.49 & 8.85 & 2.78 & 1.36 & 1.42 & MS, RI \\
\hline 2 & $\alpha$-pinene & 944 & 939 & 1.16 & 2.90 & 1.58 & 0.82 & 0.25 & 0.49 & MS, RI, Std. \\
\hline 3 & sabinene & 977 & 973 & 0.48 & 1.19 & 0.96 & $--^{\mathrm{d}}$ & - & - & MS, RI, Std. \\
\hline 4 & $\beta$-pinene & 981 & 980 & 1.12 & 2.78 & 2.06 & - & - & - & MS, RI, Std. \\
\hline 5 & $p$-cymene & 1022 & 1026 & 11.55 & 17.97 & 27.74 & 7.28 & 3.50 & 7.59 & MS, RI, Std. \\
\hline 6 & limonene & 1028 & 1031 & 0.72 & 1.81 & 1.27 & - & - & - & MS, RI, Std. \\
\hline 7 & $\gamma$-terpinene & 1056 & 1062 & 0.37 & 1.76 & 0.52 & - & - & - & MS, RI, Std. \\
\hline 8 & cis-4-methoxythujane & 1092 & 1088 & 0.36 & 0.97 & 0.58 & 0.99 & 0.46 & 0.63 & MS, RI \\
\hline 9 & trans-4-methoxythujane & $1117 / 1116$ & 1110 & 1.79 & 4.01 & 3.55 & 6.55 & 3.71 & 4.81 & MS, RI \\
\hline 10 & 4-terpineol & $1180 / 1178$ & 1189 & 0.16 & 0.59 & 0.28 & 1.37 & 0.86 & 1.25 & MS, RI, Std. \\
\hline 11 & dihydrocarvone & 1196 & 1193 & 0.06 & 0.80 & 0.49 & 0.86 & 0.57 & 0.79 & MS, RI, Std. \\
\hline 12 & thymoquinone & 1247 & 1249 & 9.37 & 32.95 & 39.73 & 29.83 & 47.39 & 50.62 & MS, RI, Std. \\
\hline 13 & $(E)$-dec-2-enal & 1260 & 1261 & 0.17 & 0.22 & 0.02 & - & - & - & MS, RI, Std. \\
\hline 14 & thymol & $1294 / 1293$ & 1290 & 0.59 & 0.41 & 0.95 & 2.76 & 1.59 & 4.82 & MS, RI, Std. \\
\hline 15 & $(E, E)$-deca-2,4-dienal & 1314 & 1314 & 0.64 & 0.55 & 0.16 & - & - & - & MS, RI, Std. \\
\hline 16 & tetradecane & 1400 & 1400 & 0.18 & 0.50 & 0.07 & - & - & - & MS, RI, Std. \\
\hline 17 & longifolene & 1403 & 1402 & 0.30 & 2.53 & 1.35 & - & - & - & MS, RI \\
\hline 18 & thymohydroquinone & $1551 / 1552$ & 1559 & 0.77 & 0.61 & 0.55 & 13.65 & 10.80 & 13.12 & MS, RI, Std. \\
\hline 19 & 2,4-diethoxybenzoic acid & $1724 / 1726$ & n.a. ${ }^{e}$ & 0.21 & 0.20 & 0.05 & 5.61 & 1.66 & 1.24 & MS, Std. \\
\hline 20 & 3,7-dimethyloctanal & 1729 & n.a. ${ }^{e}$ & - & - & - & 4.13 & 3.79 & 0.33 & MS, Std. \\
\hline 21 & hexadecanoic acid & $1960 / 1958$ & 1970 & 8.92 & 0.91 & 1.42 & 1.68 & 0.58 & 0.26 & MS, RI, Std. \\
\hline 22 & sesquiterpene ester ${ }^{\mathrm{f}}$ & $2128 / 2130$ & n.a. ${ }^{e}$ & 1.68 & 6.03 & 0.07 & 6.36 & 2.22 & 5.31 & MS \\
\hline 23 & octadecadienoic acid & $2153 / 2159$ & 2150 & 32.86 & 5.5 & 4.55 & 6.64 & 5.44 & 1.50 & MS, RI, Std. \\
\hline 24 & octadecenoic acid & 2163 & 2161 & 21.63 & 4.32 & 3.2 & 4.03 & 3.55 & 1.97 & MS, RI, Std. \\
\hline 25 & $\beta$-monolinolein & - & n.a. ${ }^{\mathrm{e}}$ & - & - & - & 4.66 & 12.27 & 3.85 & MS \\
\hline
\end{tabular}

aRetention indices calculated from retention times on a DB-5 column and based on $\mathrm{C}_{9}-\mathrm{C}_{29}$ alkanes; ${ }^{\mathrm{b}}$ data taken from literature; ${ }^{15,28}$ cidentification method: MS: mass spectrum was identical to that of National Institute of Standards and Technology Library (ver. 2.0.f), RI: the retention index was consistent with that of the literature database, Std.: constituent identity confirmed by co-injection of authentic standards; ${ }^{\mathrm{d}}$ compound not determined in the sample; eliterature data not available; ${ }^{\mathrm{f}}$ tentatively identified compound. Data on quinone compounds are indicated in bold.

antimicrobial effects). ${ }^{29}$ Clinical studies ${ }^{30}$ proved the antifungal effect of Trachyspermum ammi essential oils, where thymol, $p$-cymene and $\gamma$-terpinene were found as the most abundant constituents. Assays with animals demonstrated that $p$-cymene has analgesic and antiinflammatory effects..$^{31}$ It is possible that these compounds will contribute to biological effects of the final product. Typical chromatogram of the extract obtained by extraction by $70 \%$ ethanol from crude oil of $N$. sativa seeds is shown in the Figure 3.

Because of its significant biological effects, TQ has been extracted by different methods from $N$. sativa seeds. ${ }^{6}$ However, the currently used effective extraction methods are expensive or they rely on toxic organic solvents. ${ }^{16,17}$ In contrast, the cheap and simple methods usually provide low yield of TQ in the final product. ${ }^{13}$ The advantage of our method is that no thermal degradation of components occurs during extraction as in the case of hydrodistillation and steam distillation, whereas the expensive equipment (e.g. SFE) is not needed. Moreover, the only organic solvent used for extraction of $N$. sativa crude oil (usually obtained by cold pressing of seeds in yield ranging from 22 to $25 \%)^{32}$ is the ethanol that is generally accepted for many food and pharmaceutical applications and products as it ensures efficiency and it is greener when compared to conventional ones. ${ }^{33}$ Nevertheless, the direct extraction of seeds by ethanol will not provide comparable results since the content of TQ is significantly lower (up to $0.03 \%),{ }^{34}$ whereas the extract consists mainly of fatty acids accompanied by broth spectrum of various compounds 


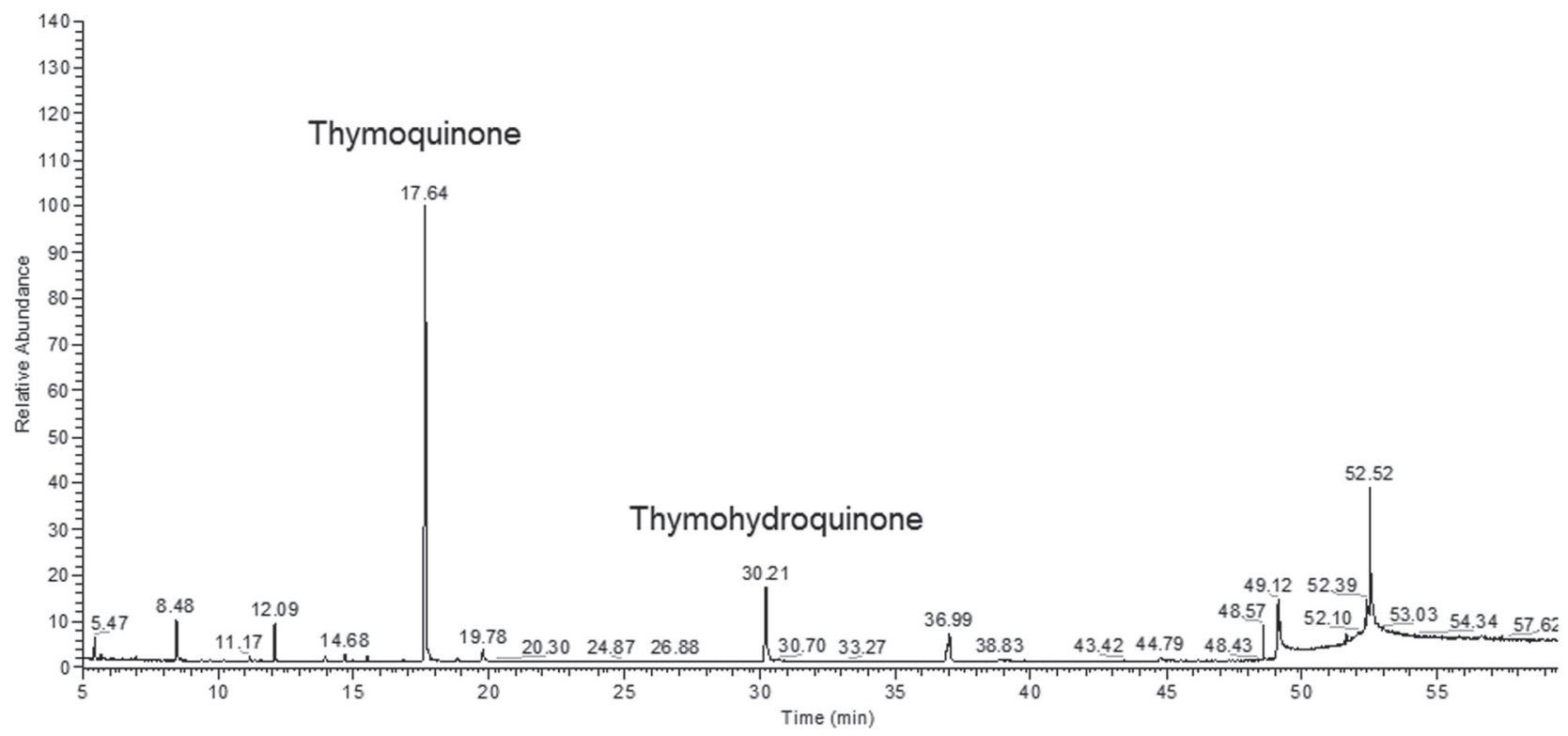

Figure 3. Chromatogram of the extract obtained by $70 \%$ ethanol from crude oil of $N$. sativa seeds.

including flavanoids, saponins, and tannins. ${ }^{35}$

\section{Conclusions}

In this article, we describe a new, fast, inexpensive and ecological liquid-liquid separation method for preparing quinones enriched extract from $N$. sativa seed oil. Method of extraction consists of several steps, including preparation of $70 \%$ solution ethanol in water, shaking the solution with $N$. sativa crude oil and separation of two clearly visible layers. As a result of this process, the top layer enriched with TQ and THQ is obtained. This chemically defined extract has a potential for the use in food, pharmaceutical and cosmetic industries, whereas method of its preparation is suitable for laboratory and industrial-scale applications. Fastness, simplicity, extraction efficiency, economic modesty and environmental friendliness are the main advantages of the method.

\section{Acknowledgments}

This research was supported by the Internal Grant Agency of the Czech University of Life Sciences Prague (project No. IGA 20185019) and Institute of Organic Chemistry and Biochemistry of the Academy of Sciences of the Czech Republic (subvention for development of research organization RVO: 61388963).

\section{References}

1. Chemat, F.; Vian, M. A.; Cravotto, G.; Int. J. Mol. Sci. 2012, 13,8615 .
2. Herrero, M.; Cifuentes, A.; Ibanez, E.; Food Chem. 2006, 98 , 136.

3. Bart, H. J.; Pilz, S.; Industrial Scale Natural Products Extraction; Wiley-VCH Verlag GmbH \& Co. KGaA: Weinheim, Germany, 2011.

4. Ipor, I. B.; Oyen, L. P. A. In Plant Resources of Southeast Asia 13; Guzman, C. C.; Siemonsma J. S., eds.; Backhuys Publishers: Leiden, Netherlands, 1999, p. 148.

5. Liu, X.; Abd El-Aty, A. M.; Shim, J. H.; Mini-Rev. Med. Chem. 2011, 11, 947.

6. Kokoska, L.; Chemistry and Biological Activity of Nigella Genus: The Antimicrobial and Anti-Inflammatory Effects of Seed Extracts, Essential Oils and Compounds of Six Nigella Species; LAP LAMBERT Academic Publishing: Saarbrucken, Germany, 2011.

7. Goyal, S. N.; Prajapati, C. P.; Gore, P. R.; Patil, C. R.; Mahajan, U. B.; Sharma, C.; Talla, S. P.; Ojha, S. K.; Front. Pharmacol. 2017, 8, article number 656.

8. Akhondian, J.; Kianifar, H.; Raoofziaee, M.; Moayedpou, A.; Toosi, M. B.; Khajedaluee, M.; Epilepsy Res. 2011, 93, 39.

9. Kapil, H.; Suresh, D. K.; Bathla, S. C.; Arora, K. S.; Saudi Dent. J. 2018, 30, 348.

10. Toama, M. A.; El-Alfy, T. S.; El-Fatatry, H. M.; Antimicrob. Agents Chemother. 1974, 6, 225; Halamova, K.; Kokoska, L.; Flesar, J.; Sklenickova, O.; Svobodova, B.; Marsik, P.; J. Food Prot. 2010, 73, 2291; Marsik, P.; Kokoska, L.; Landa, P.; Nepovim, A.; Soudek, P.; Vanek, T.; Planta Med. 2005, 71, 739; Ivankovic, S.; Stojkovic, R.; Jukic, M.; Milos, M.; Milos, M.; Jurin, M.; Exp. Oncol. 2006, 28, 220.

11. Tesarova, H.; Svobodova, B.; Kokoska, L.; Marsik, P.; Pribylova, M.; Landa, P.; Vadlejch, J.; Nat. Prod. Commun. 2011, 6, 213. 
12. Jukic, M.; Politeo, O.; Maksimovic, M.; Milos, M.; Phytother. Res. 2007, 21, 259.

13. Benkaci-Ali, F.; Baaliouamer, A.; Meklati, B. Y.; Chemat, F.; Flavour Fragrance J. 2007, 22, 148; Gerige, S. J.; Gerige, M. K. Y.; Rao, M.; Ramanjaneyulu, X.; Braz. Arch. Biol. Technol. 2009, 52, 1189.

14. Kokoska, L.; Havlik, J.; Valterova, I.; Sovova, H.; Sajfrtova, M.; Jankovska, I.; J. Food Prot. 2008, 71, 2475.

15. Wajs, A.; Bonikowski, R.; Kalemba, D.; Flavour Fragrance J. 2008, 23, 126.

16. Taborsky, J.; Kunt, M.; Kloucek, P.; Lachman, J.; Zeleny, V.; Kokoska, L.; Cent. Eur. J. Chem. 2012, 10, 1899.

17. Piras, A.; Rosa, A.; Marongiu, B.; Porcedda, S.; Falconieri, D.; Dessi, M. A.; Ozcelik, B.; Koca, U.; Ind. Crops Prod. 2013, 46, 317.

18. Brasero, N.; Martinet, B.; Lecocq, T.; Lhomme, P.; Biella, P.; Valterova, I.; Urbanova, K.; Cornalba, M.; Hines, H.; Rasmont, P.; Insect Sci. 2018, 25, 75.

19. Yalkowsky, S. H.; He, Y.; Jain, P.; Handbook of Aqueous Solubility Data, $2^{\text {nd }}$ ed.; CRC Press: Boca Raton, USA, 2010.

20. Mouhajir, F.; Pedersen, J. A.; Rejdali, M.; Towers, G. H. N.; Pharm. Biol. 1999, 37, 391.

21. Durling, N. E.; Catchpole, O. J.; Grey, J. B.; Webby, R. F.; Mitchell, K. A.; Foo, L. Y.; Perry, N. B.; Food Chem. 2007, 101, 1417.

22. Mokrani, A.; Madani, K.; Sep. Purif. Technol. 2016, 162, 68.

23. Casazza, A. A.; Aliakbarian, B.; Perego, P.; Nat. Prod. Res. 2011, 25, 1751.

24. Naczk, M.; Shahidi, F.; J. Pharm. Biomed. Anal. 2006, 41, 1523.

25. Liu, X.; Abd El-Aty, A. M.; Cho, S. K.; Yang, A.; Park, J. H.; Shim, J. H.; Biomed. Chromatogr. 2012, 26, 1157.

26. Nickavar, A.; Mojab, F.; Javidnia, K.; Amoli, M. A. R.; Z. Naturforsch., C: J. Biosci. 2003, 58, 629.

27. Hamrouni-Sellami, I.; Kchouk, M. E.; Marzouk, B.; J. Food Biochem. 2008, 32, 335.
28. Adams, R. P.; Identification of Essential Oil Components by Gas Chromatography/Mass Spectrometry, $4^{\text {th }}$ ed; Allured Publishing Corporation: Carol Stream, USA, 2007; Linstrom, P. J.; Mallard, W. G.; NIST WebBook Chemistry, NIST Standard Reference Database Number 69; National Institute of Standards and Technology: Gaithersburg, USA, 2017.

29. Bakkali, F.; Averbeck, S.; Averbeck, D.; Idaomar, M.; Food Chem. Toxicol. 2008, 46, 446; Kalemba, D.; Kunicka, A.; Curr. Med. Chem. 2003, 10, 813; Ruberto, G.; Baratta, M. T.; Food Chem. 2000, 69, 167.

30. Sharifzadeh, A.; Khosravi, A. R.; Shokri, H.; Sharafi, G.; J. Mycol. Med. 2015, 25, 143.

31. Quintans, J. D. S.; Menezes, P. P.; Santos, M. R. V.; Bonjardim, L. R.; Almeida, J. R. G. S.; Gelain, D. P.; Araujo, A. A. D.; Quintans, L. J.; Phytomedicine 2013, 20, 436.

32. Mazaheri, Y.; Torbati, M.; Azadmard-Damirchi, S.; Savage, G. P.; Food Chem. 2019, 274, 480; Atta, M. B.; Food Chem. 2003, 83,63 .

33. Hron, R. J. In Technology and Solvents for Extracting Oilseeds and Nonpetroleum Oils; Wan, P. J.; Wakelyn, P. J., eds.; AOCS Press: Champaign, USA, 1997, p. 192; Bellete, B. S.; Ramin, L. Z.; Porto, D.; Ribeiro, A. I.; Forim, M. R.; Zuin, V. G.; Fernandes, J. B.; Silva, M. F. G. F.; J. Braz. Chem. Soc. 2018, 29, 1123.

34. Nivetha, K.; Prasanna, G.; Int. J. Adv. Res. Biol. Sci. 2016, 3 , 45; Mohamed, M. S. N.; Jaikumar, K.; Marimuthu, S.; Wyson, W. J.; Anand, D.; Periasamy, S.; Asian J. Pharm. Clin. Res. 2017, 10, 329.

35. Dwarampudi, L. P.; Palaniswamy, D.; Nithyanantham, M.; Raghu, P. S.; Pharmacogn. Mag. 2012, 8, 268; Ishtiaq, S.; Ashraf, M.; Hayat, M. Q.; Asrar, M.; Int. J. Agric. Biol. 2013, 15,1511 .
Submitted: January 30, 2019

Published online: October 8, 2019 